were no significant differences in the TSH and 17-OHP values in patients and controls. In a separate analysis of 6 infants exposed to polytherapy of 2 or more AEDs, there was a non-significant tendency to lower TSH and 17-OHP. Thyroid and steroid screening values were not correlated with AED plasma concentrations measured 1 month before delivery. (Wide $\mathrm{K}$ et al. Antiepileptic drug treatment during pregnancy and neonatal screening results. Acta Paediatr July 1996;85:870-1). (Respond: Dr K Wide, Department of Paediatrics, Karolinska Hospital, S-171 76 Stockholm, Sweden).

COMMENT. Carbamazepine and phenytoin induce metabolic enzymes and enhance metabolism of steroid and thyroid hormones. Low levels of thyroxine T4 and steroid hormones are reported in young adults treated with CBZ and PHT but not with valproate monotherapy. Serum T3 is unaffected by AEDs. (Prog Ped Neur I, 1991, pp126-7). The above study suggests that CBZ and PHT monotherapy during pregnancy may not alter fetal thyroid and steroid metabolism.

\title{
VALPROIC ACID AND THROMBOCYTOPENIA
}

The association of thrombocytopenia (TCP) with valproic acid (VPA) therapy was evaluated retrospectively in 167 children treated with VPA between 1989 and 1993 at the Department of Pediatrics, Henry Ford Medical Center, Detroit, MI. VPA monotherapy in 91 and VPA polytherapy in 76 children were compared with 92 age- and sex-matched controls taking AEDs other than VPA. Thrombocytopenia $\left(<200 \times 10^{3} / \mathrm{mm}^{3}\right)$ occurred in $22 \%$ of VPA treated children (in $26 \%$ on monotherapy and $16 \%$ on polytherapy), and in $5 \%$ of controls. Patients with TCP were older, had higher serum VPA levels, and received higher doses of VPA than those without TCP. The degree of TCP was mild, no patient developed bleeding or excess bruising, and VPA was not discontinued because of TCP. (Allarakhia IN, Garofalo EA, Komarynski MA, Robertson PL. Valproic acid and thrombocytopenia in children: a casecontrolled retrospective study. Pediatr Neurol May 1996;14:303-7). (Respond: Dr I Allarakhia, Department of Pediatrics, Henry Ford Medical Center, 2799 West Grand Boulevard, Detroit, MI 48202).

COMMENT. Despite this documentation of a $22 \%$ incidence of thrombocytopenia in children treated with VPA, severe TPA with bleeding complications did not occur, and the withdrawal of VPA was not required. The authors recommend close monitoring of the platelet count in patients receiving VPA in larger doses and with higher serum levels, and particularly in older children. VPA induced bleeding may sometimes be explained by an underlying familial disease, eg. von Willebrand pseudohemophilia or a dysfibrinogenemia. (see Progress in Pediatric Neurology II, 1994, pp102-103).

Pseudo valproate-induced hypofibrinogenemia is reported in a 6-year-old boy with a ventriculoperitoneal shunt for hydrocephalus and a stone in the ureter requiring surgery at the Departments of Pediatrics and Neurology, Park Nicollet Clinic, Minneapolis, MN. Pre-surgical coagulation studies revealed a prothrombin time of $14.9 \mathrm{~s}(\mathrm{~N} \mathrm{9.8-13.2),} \mathrm{thrombin} \mathrm{time} \mathrm{of}$ $73.3 \mathrm{~s}$ (N 13-20), and fibrinogen levels of $<50 \mathrm{mg} / \mathrm{dl}$ (N 145-375). The patient's mother also had a prolonged thrombin time, and a diagnosis of inherited dysfibrinogenemia was presumed in this case. (Breningstall GN, Cich JA. Pediatr Neurol May 1996;14:345). Two previous reports are cited of a valproate dose-related decrease in fibrinogen, and one neonate whose mother was receiving VPA had a symptomatic fibrinogen deficiency. 